\title{
ADHESIVE REATTACHMENT OF A PREMOLAR TOOTH FRAGMENT REINFORCED WITH POLYETHYLENE FIBÉRS-A CASE REPORT.
}

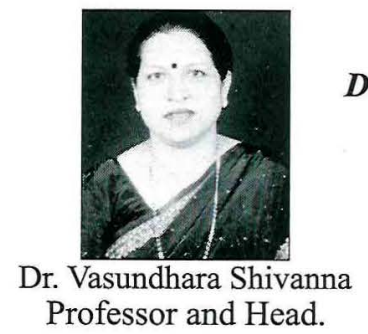

Recent developments in restorative materials, placement techniques, preparation design, and adhesive protocols allow clinicians to predictably restore fractured teeth. Using a minimally invasive approach, treatment of fractured teeth can be completed. If the original tooth fragment is retained following fracture, the natural tooth structures can be reattached using adhesive protocols to ensure liable strength, durability, and aesthetics. ${ }^{1}$

Several factors influence the management of coronal tooth fractures, including extent of fracture (biological width violation, endodontic involvement, alveolar bone fracture), pattern of fracture and restorability of fractured tooth (associated root fracture), secondary trauma injuries (soft tissue status), presence/absence of fractured tooth fragment and its condition for use (fit between fragment and the remaining tooth structure), occlusion, esthetics, finances, and prognosis. Patient cooperation and understanding of the limitations of the treatment is of utmost importance for good prognosis. ${ }^{2}$

The restoration of the pulpless tooth is a critical final step of successful endodontic therapy. Emphasis has been placed on intracoronal strengthening of teeth to protect them against fracture, but controversy exists still regarding the preferred type of final restoration. When a cuspal fracture occurs, the tooth can be restored by reattaching the fragment to the remaining tooth structure, using a dentine bonding system and a resin composite. Only a few in vitro studies report the success of such restorations in laboratory conditions. ${ }^{3}$

This purpose of this article is to discuss a case of adhesive reattachment of a tooth fragment to a fractured premolar tooth after endodontic treatment and reinforced with polyethylene fibers.

Case report: A 25 years old male reported to the department of conservative dentistry and endodontics, college of dental sciences with a chief complaint of pain with respect to right upper back tooth region. Patient gave a history of trauma.

Clinically: Maxillary right first premolar showed fracture of lingual cusp (Fig.1) with the fracture line extending from central groove, subgingivally to detach the lingual cusp from the rest of the tooth. The fragment remained attached to the sub-gingival tissues.

Treatment: Since the fracture line was extending close to the pulp and was as well extending sub-gingivally, it was decided to complete root canal treatment and then reattach the fragment after palatal flap reflection.

Before beginning the root canal treatment, the tooth fragment was extracted and placed in saline until fragment was reattached. Single sitting root canal treatment was done. A sulcular incision was placed extending from mesial of canine to distal of second premolar. Flap was reflected to expose the extent of fracture line (Fig.2). After hemostasis was achieved, tooth was etched for 15 secs using $37 \%$ phosphoric acid (Scotchbond etchant; 3M ESPE) and was thoroughly rinsed using water for 10secs. Tooth was dried using small cotton pellets following which dentine bonding agent (XP Bond; Universal total-etch adhesive, Dentsply) was applied, gently air dried and cured for 10 secs. Simultaneously the tooth fragment was also etched, rinsed with water and dried. 
Dentine bonding agent was applied to the tooth fragment and cured extra-orally similar to that done on the tooth structure. Flowable composite resin (Filtek ${ }^{\mathrm{TM}} \mathrm{Z} 350$ Flowable restorative; 3M ESPE) was applied on the fragment and tooth structure, fragment was then approximated to its position on the tooth and was cured for 20 secs from all directions. The access cavity was restored using composite resin (Filtek ${ }^{\mathrm{TM}} \mathrm{Z} 350 \mathrm{XT}$ Universal Restorative; 3M ESPE) and cured for 40secs. Flap was re-approximated and sutured. Appropriate occlusal adjustments were made.

The patient was followed after a week, sutures were removed (Fig.3) and a groove was prepared bucco-lingually of $2 \mathrm{~mm}$ width and depth (Fig.4). A best fit of Polyethylene fibers (Ribbond THM, Ribbond) was cut (Fig.5). Since these fibers are not pre-impregnated with resin the fibers were coated with a thin layer of dentine bonding agent (XP Bond; Dentsply) and cured. The fiber was inserted in the groove (Fig.6); thin layer of flowable resin was applied and cured for 40 secs. The groove was then restored using composite resin (Fig.7). Finishing and polishing of the restoration was done using Shofu composite polishing kit. A follow up was done after $3 \frac{1}{2}$ months (Fig.8). The tooth was seen to be functioning without any complications.

\section{Discussion:}

Restoration of the teeth is an important final step of endodontic treatment. Previous studies have indicated that full cast crown restorations with or without post placement, an indirect cast restoration covering the cusps (onlay), complex amalgam restorations, or composite materials can be used for final restoration. Post placement may result in weakening of roots. ${ }^{4}$ Full cast crowns and other indirect cast restorations result in considerable loss of tooth structure, are expensive and time consuming. ${ }^{5}$ When restoration margins are to be placed especially subgingivally, problems for the dentist is both technical and biological in nature, i.e., the technical difficulties related to the impression-taking and the biological risk of further damaging the periodontal tissues. Even if a well-adapted restoration is fabricated, studies have reported that subgingivally placed restorations always result in an inflammatory periodontaliresponse. ${ }^{6}$

One of the options for managing coronal tooth fractures, especially when there is no or minimal violation of the biological width, is the reattachment of the dental fragment when it is available. Tooth fragment reattachment offers a conservative, esthetic, and cost-effective restorative option that has been shown to be an acceptable alternative to the restoration of the fractured tooth with resin-based composite or full-coverage crown. Reattachment of a fragment to the fractured tooth can provide good and long-lasting esthetics, can restore function, can result in a positive psychological response, and is a reasonably simple procedure. In addition, tooth fragment reattachment allows restoration of the tooth with minimal sacrifice of the remaining tooth structure. Furthermore, this technique is less time-consuming and provides a more predictable long-term wear than when direct composite is used. Clinical trials and longterm follow-up have reported that reattachment using modern dentin-bonding agents or adhesive luting systems may achieve functional and esthetic success. $^{2}$

Shrinkage of composite materials during polymerization and its stresses generated are one of the prime factors that adversely affect the success of direct composite restoration which causes microcracking in the bulk, weakening of interfaces, and even debonding of local areas between bonded surfaces. One of the suggested methods for reducing debonding during polymerization shrinkage is the application of a low viscosity, low modulus intermediate resin between the bonding agent, and restorative resin to act as an "elastic buffer" or "stress breaker" that can relieve contraction stresses and improve marginal integrity. ${ }^{3}$ Hence, in this study flowable composite resin was used to attach tooth fragment.

It is advisable not to use the simple reattachment technique without additional preparation as this technique may not be able to restore sufficient fracture strength. It is desirable to use any of the reinforcement techniques so as to increase the fracture strength of the reattached tooth fragment, thereby improving the longevity of the treated tooth.? 
The presence of fiber network creates a change in stress dynamics due to its higher modulus of elasticity and lower flexural modulus which may have a modifying effect on how the interfacial stresses will develop along the restoration / tooth interface. Two of the mechanisms by which fibers exert high strain to failure on brittle composite matrix are by acting as stress-bearing component and by acting as crackstopping or crack-deflecting mechanisms. ${ }^{8}$ Placing fibers at the occlusal $3^{\text {rd }}$ extending their ends from buccal to lingual walls allows the fibers to keep the cusps together, resulting in better fracture strength ${ }^{4}$ and hence, reinforcing the tooth.

Conclusion: Reattachment of the fractured tooth fragment is the most conservative and biological method of restoring a fractured tooth. However, many studies are required to predict its long term success rate.

\section{References:}

1. Terry D A. Adhesive reattachment of a tooth fragment: The biological restoration. Pract Proced Aesthet Dent. 2003; 15(5): 403-9.

2. Macedo G V, Diaz P I, Fernandes C A, Ritter A V. Reattachment of anterior teeth fragments: a conservative approach. J Esthet Restor Dent 2008; 20(1): 5-20.
3. Belli S, Cobankara F K, Eraslan O, Eskitascioglu G, Karbhari V. The effect of fiber insertion on fracture resistance of endodontically treated molars with MOD cavity and reattached fractured lingual cusps. J Biomed Mater Res Part B: Appl Biomater 2006; 79B: 35-41.

4. Sengun A, Cobankara F K, Orucoglu H. Effect of a new restoration technique on fracture resistance of endodontically treated teeth. Dent Traumatol 2008; 24: 214-9.

5. Uyehara M Y, Davis R D, Overton J D. Cuspal reinforcement in endodontically treated molars. Oper Dent 1999; 24: 36470.

6. Hansen E K. In vivo cusp fracture of endodontically treated premolars restored with MOD amalgam or MOD resin fillings. Dent Mater 1988; 4(4): 169-73.

7. Goenka P, Marwah N, Dutta S. Biological approach for management of anterior tooth trauma: Triple case report. J Indian Soc Pedod Prev Dent 2010; 28(3): 223-9.

8. Rudo D N, Karbhari V M. Physical behaviors of fiber reinforcement as applied to tooth stabilization. Dent Clin N Am. 1999; 43(1): 7-35. 




Fig.1: Fractured maxillary premolar



Fig.3: 1 week post-operative

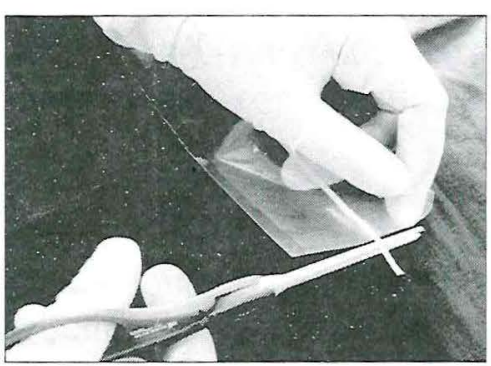

Fig.5: Polyethylene fiber (Ribbond THM)



Fig.7: Post-operative

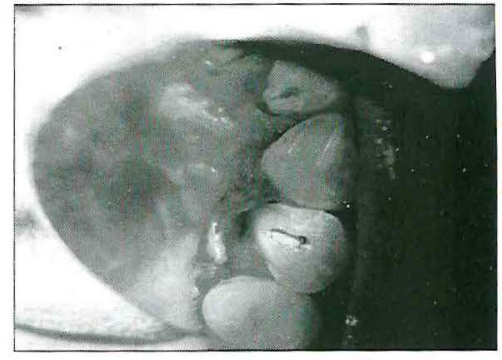

Fig.2: Reflection of flap \& extraction of tooth fragment after root canal treatment completion



Fig.4: Cavity preparation for fiber insertion

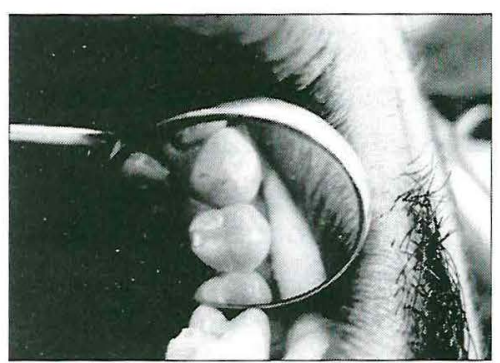

Fig.6: Fiber insertion



Fig. 8 : $31 \frac{1}{2}$ months post-operative 